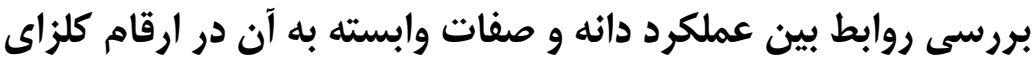 بهاره با استفاده از تجزيه عليت
}

\author{
مهدى سلطانى حويزه'، محمد مرادى"، طيب ساكىنزاد"، سعيد ذاكرنزادّ" \\ و عادل اعطآ

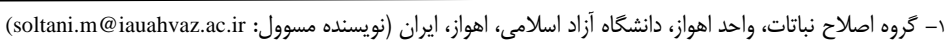

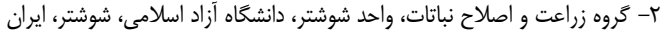

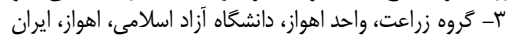

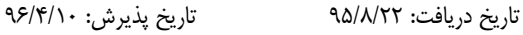

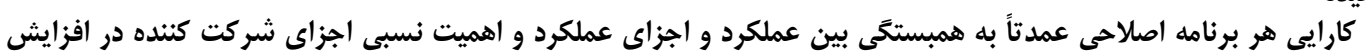

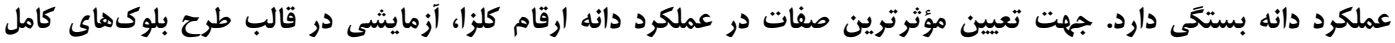

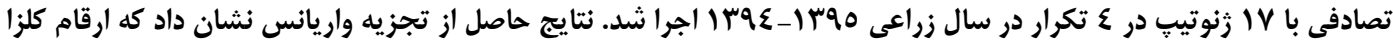

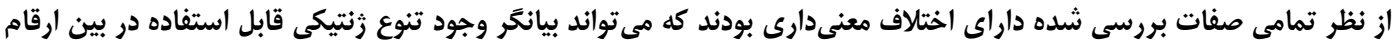

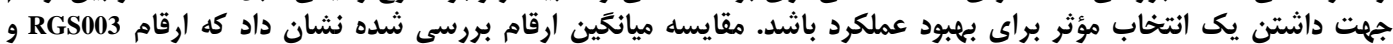

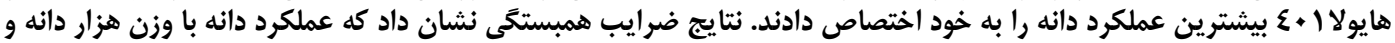

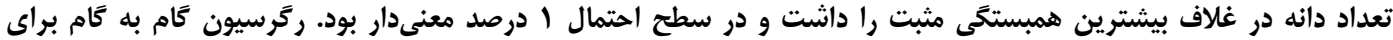

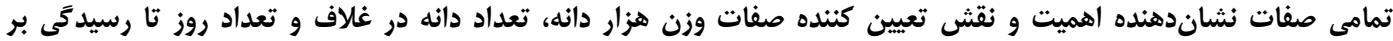

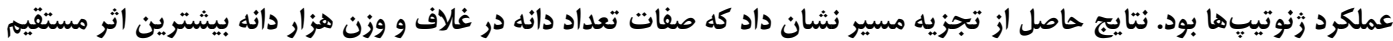

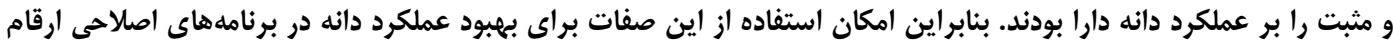
بهاره كلزا به عنوان معيار انتخاب وجود داردا بودند دارد.

وازههاى كليدى: همبستَىى، رَّرسيون كَام به كَام، تجزيه عليت و كلزا

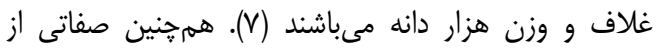

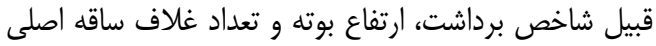

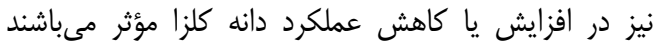

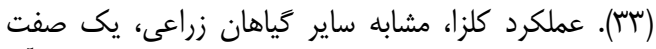

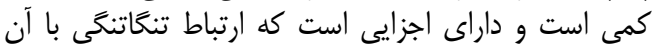

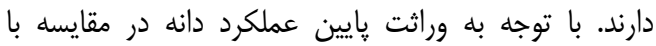

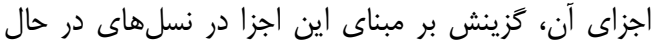

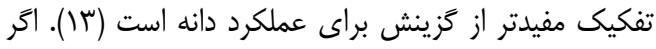

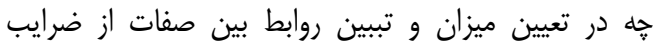

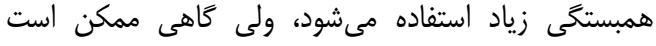

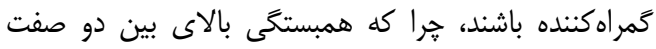

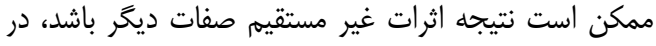

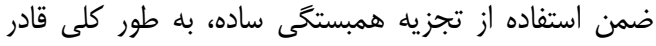

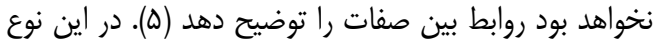

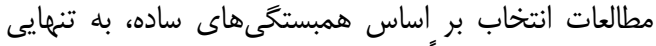

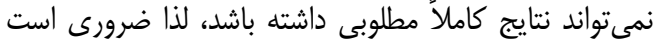

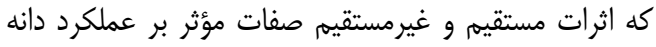

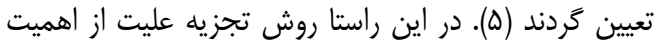

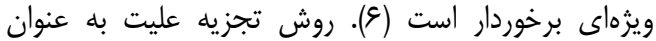

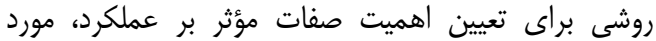

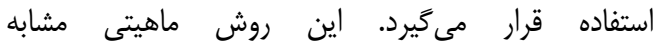

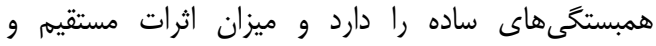

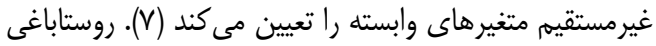

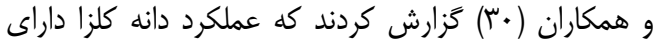

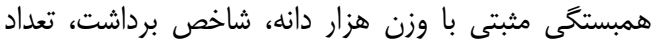

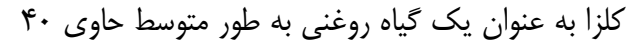

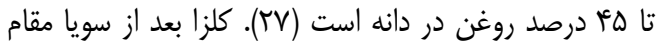

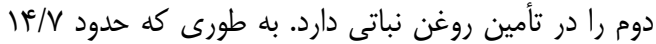

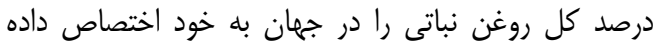

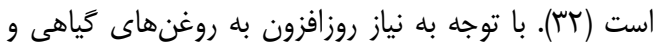

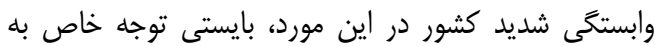

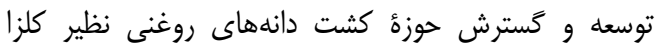

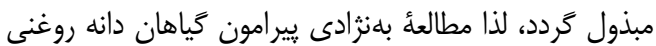

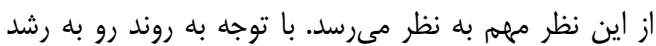

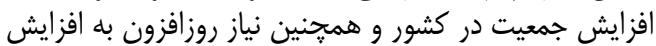

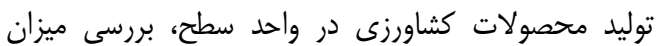

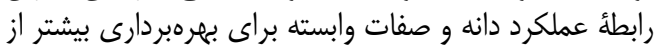

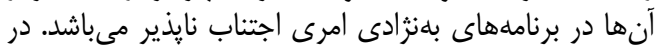

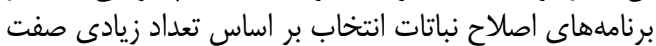

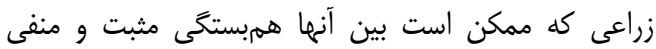

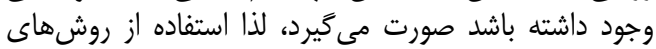

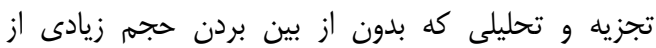

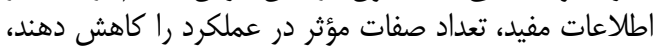

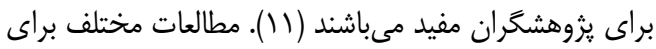

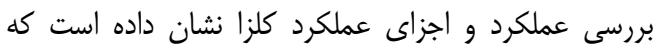

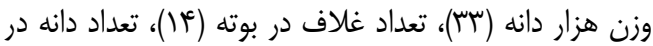

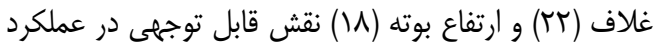

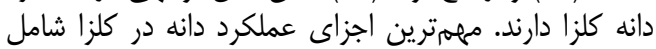

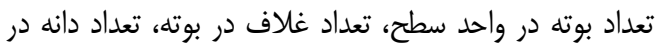




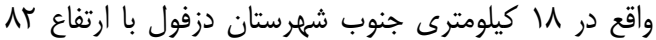

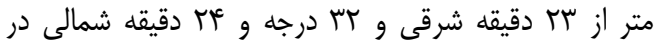

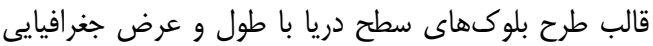

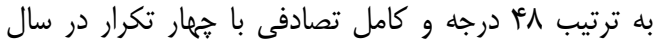

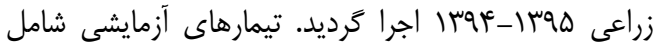

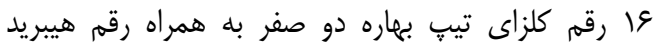

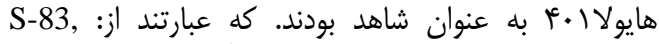
RG4403, Amica, RGAS0324, RGS006, Hyola401, Kimberley, RG405/02, RG405/03 Sarigol, Hysun110, RGS003, Hyola420, Hyola308, Hyola60, pF, Option500,

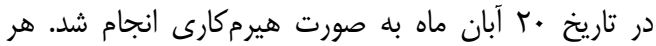
كرت آزمايشى شامل \&

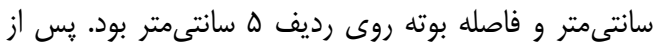

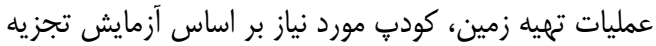

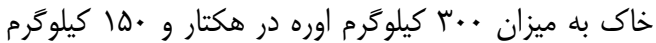

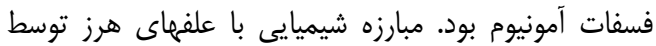

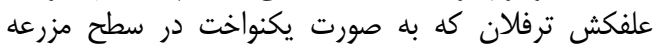

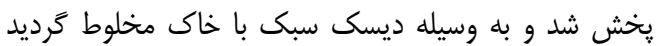

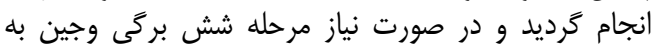

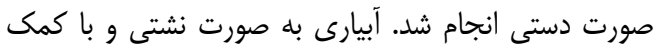

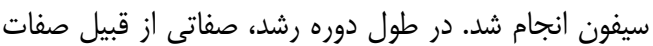

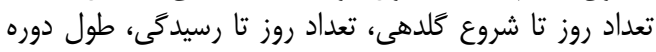

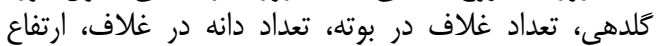

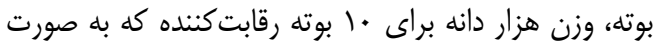

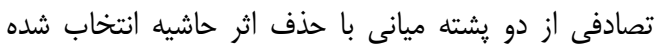

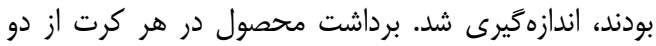

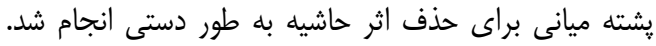

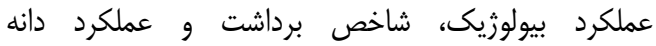

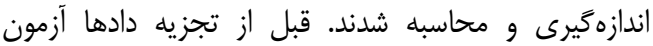
نرمال بودن دادها توسط نرمافزار آمارى

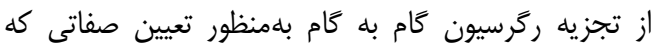

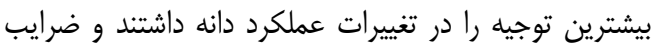

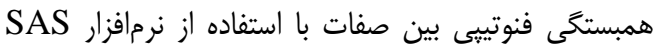

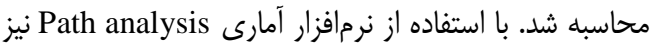

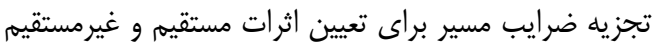
صفات بررسى شده بر عملكرد دانه استفاده شد.

\section{نتايج و بحث} تجزيه واريانس و مقايسه ميانكَين

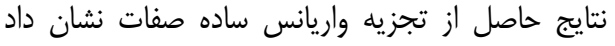

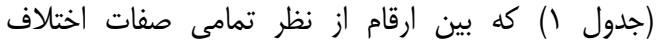

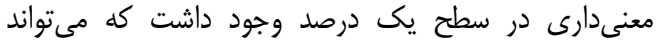

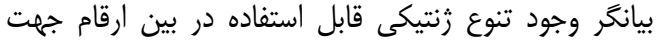

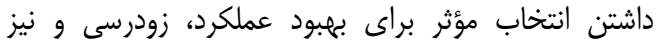

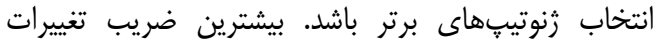

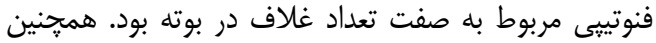

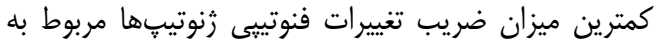

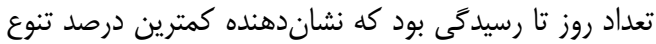

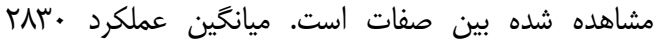

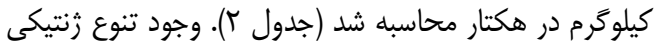

شاخه فرعى در بوته و طول دوره رسيدگى است. نتايج حاصل

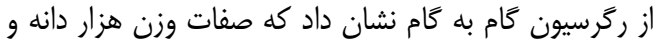

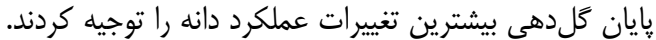

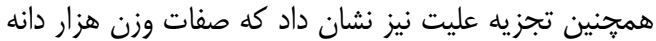

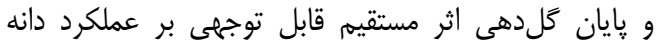

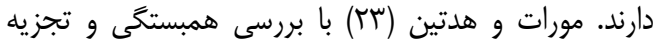

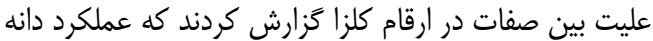

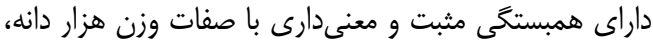

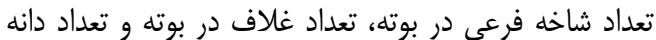

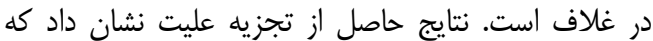

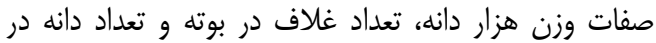

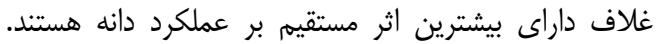

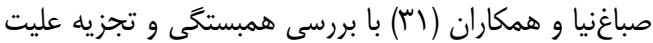

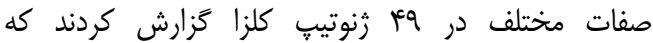

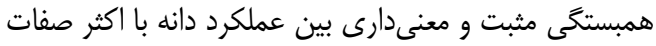

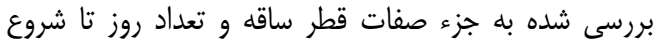

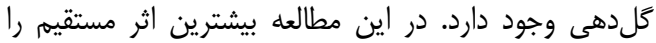

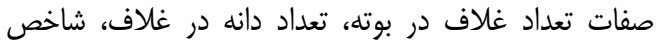

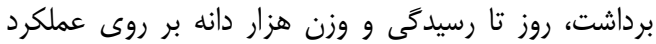

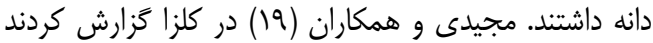

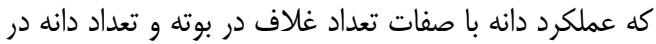

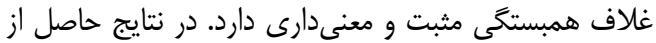

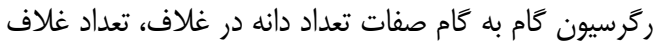

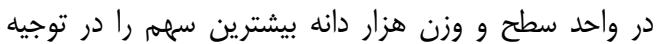

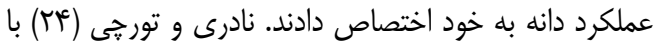

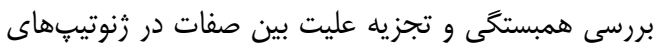

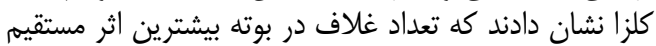

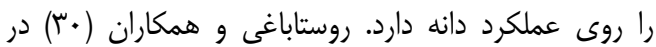

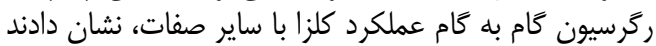

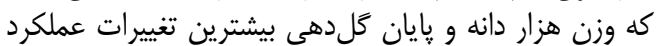

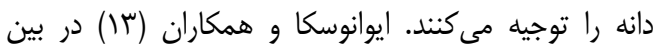

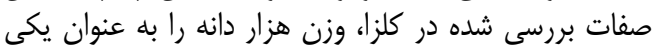

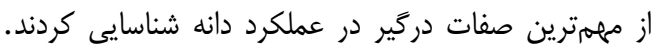

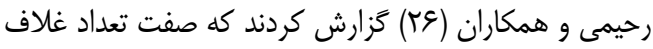

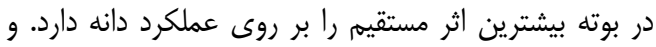

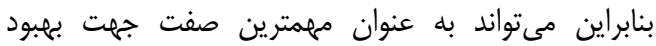

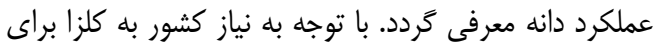

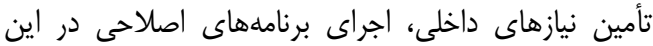

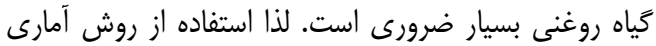

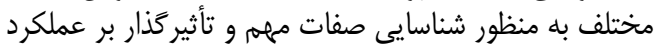

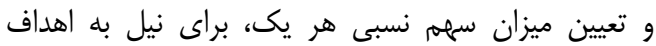

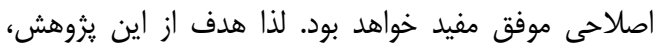

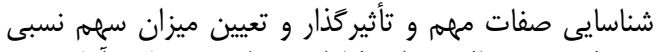

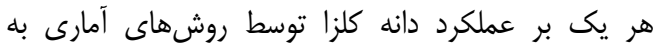
منظور استفاده در برنامههاى بانثزادى است.

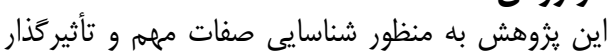

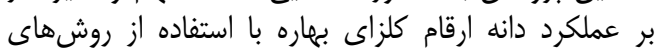

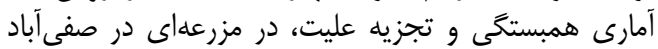


Kimberley و RGS003

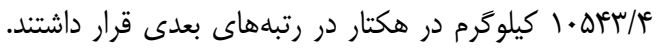
رقم Amica با متوسط

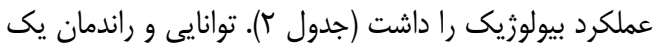

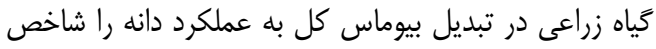

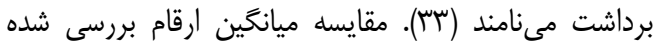

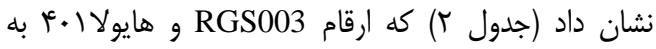

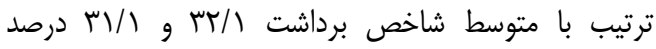

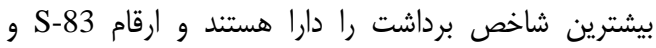

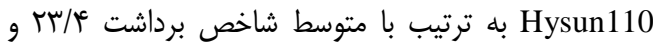

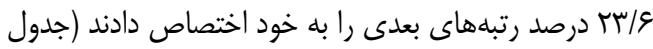

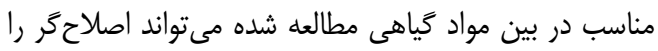

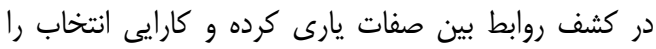

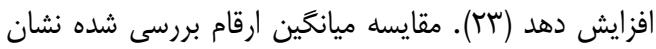

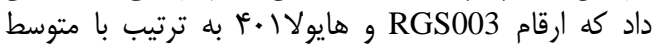

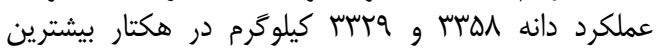

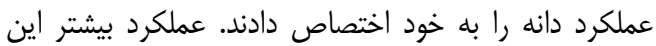

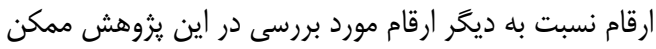

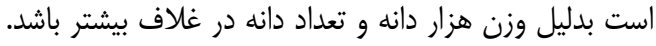

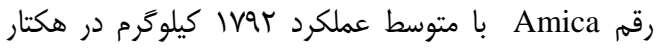

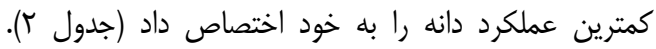

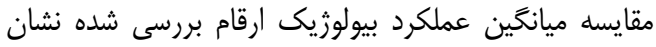

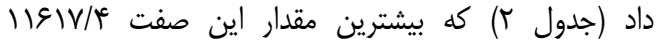

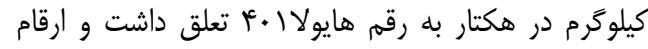

جدول ا- تجزيه واريانس عملكرد، اجزاى عملكرد و صفات فنولوزيكى در ارقام كلزا Table 1. Analysis of variance of yield, yield-componants and phenological traits in rapeseed cultivars

\begin{tabular}{|c|c|c|c|c|c|c|}
\hline \multicolumn{5}{|c|}{ ميانكَين مربعات } & \multirow[b]{2}{*}{ درجه آزادى } & \multirow[b]{2}{*}{ تنييرات } \\
\hline تعداد دانه در غلاف & بيولوزيك & تعداد غلاف & هزار دانه & عمانهرد & & \\
\hline$N / \mu^{*}$ & $r \cdot 1 r / \Lambda^{*}$ & |QTr/s & $\mid r / \Delta T^{\mathrm{ns}}$ & $q \wedge / \Gamma^{* * *}$ & r & بلوى \\
\hline$\Delta N / T f^{* * *}$ & $r \mu F \Delta / r^{* *}$ & $\Gamma \vee \wedge 9 / \Delta^{* *}$ & $\Delta r / 9 \Lambda^{* *}$ & $\operatorname{vqve} / \Lambda^{* *}$ & 18 & تيمار \\
\hline$D / \& \varphi$ & DFT/G & $\Gamma / N / r$ & s/TQ & $\Lambda \Gamma \Delta / \bar{q}$ & is & خطا \\
\hline$V /{ }^{\mu} \Lambda$ & $18 / 4 \pi$ & WNGT & $1 . / V 1$ & $\mid r / 9 T^{4}$ & & ضري \\
\hline
\end{tabular}

Continued of Table 1

ادامه جدول |

\begin{tabular}{|c|c|c|c|c|c|c|c|}
\hline \multicolumn{8}{|c|}{ ميانكين مربعات } \\
\hline تعداد روز تا & شاخص برداشت & طول دوره كلدهى & تعداد روز تا شروع & ارتفاع & روغن & درجه آزادى & تغنييرات \\
\hline$\Delta T / r^{* * *}$ & $1 \Lambda \Delta / V^{* *}$ & $1 / 10^{\mathrm{ns}}$ & $r / r \omega^{n s}$ & $\mid Q / K M^{*}$ & $r \omega / g r^{*}$ & r & بلوى \\
\hline$r \Delta / / \varphi^{* *}$ & $\mid \& Y / q^{* *}$ & q/re*** & $N / r^{* *}$ & $s \Delta / r \varepsilon^{* *}$ & १1 $/ \Delta \Lambda^{* * *}$ & 19 & تيمار \\
\hline$T / \Delta S$ & $\Delta T / I T$ & $r / \Delta V$ & T/VG & 91.9 & $V / \Delta S$ & $+\wedge$ & خطا \\
\hline$r / a V$ & $11 / \pi F$ & $\Delta / / Q$ & $r / A V$ & $\Delta / V T$ & $r / q F$ & & ضريد \\
\hline
\end{tabular}

برخورد دوره كلدهى و گرشدن دانه اين ارقام با گرماى آخر

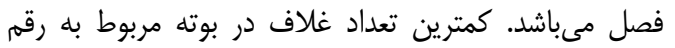

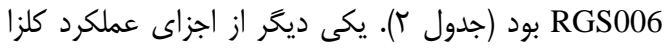

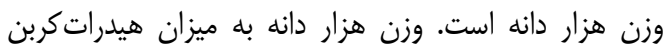

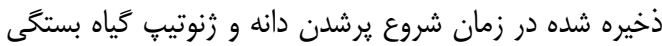

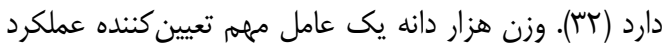

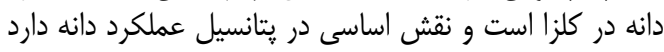

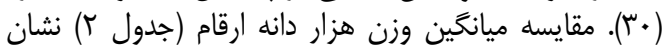

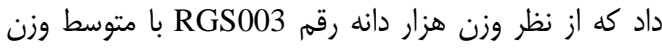

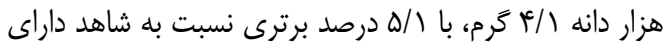

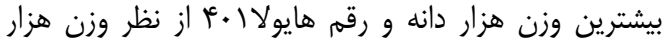

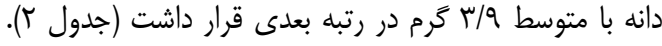

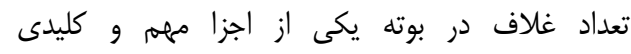

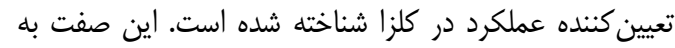

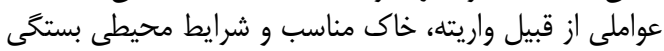

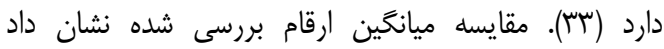

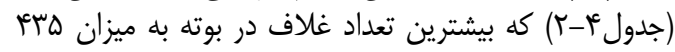

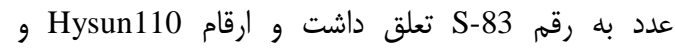

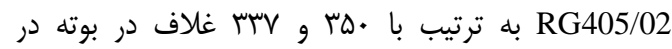

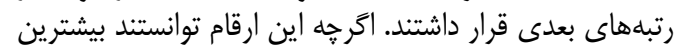

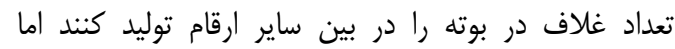

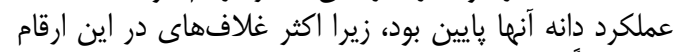

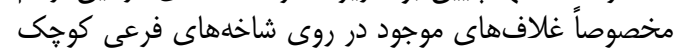

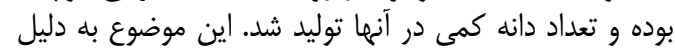




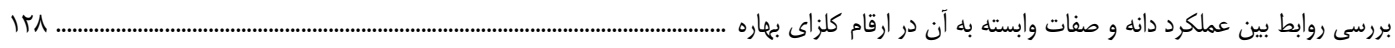

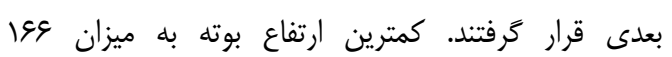

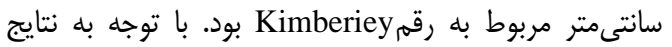

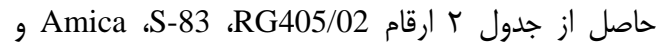
Sarigol

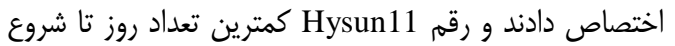

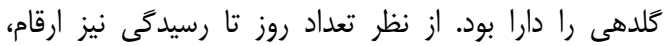
Amica ،S-83 ارقام در رتبههاى بعدى قرار داشتند. از نظر طول دوره كلدهى

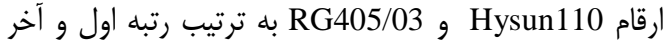

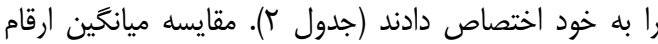

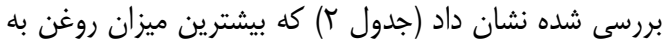

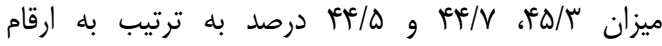

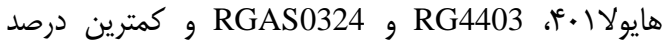
روغن به رقم S-83 تعلق داشت.

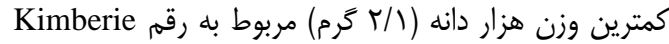

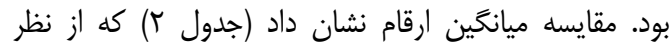

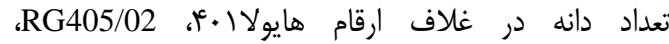
Kimberiey

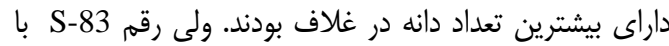
ميانخين ع دانه در غلاف داراى كمترين تعداد دانه در غاعلاف

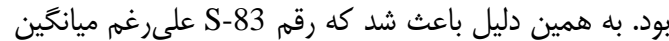

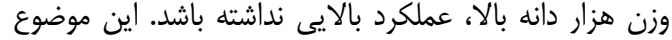

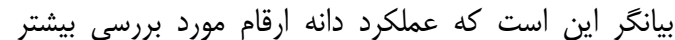

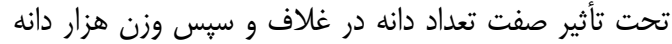

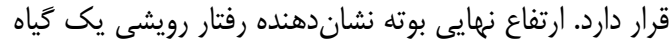

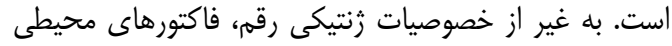

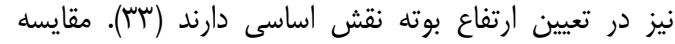

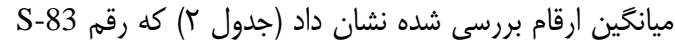

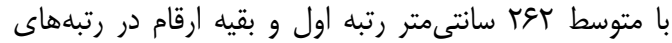


جدول r- مقايسه ميانكين عملكرد، اجزاى عملكرد و صفات فنولوزيكى در ارقام كلزا"

Table 3. Comparison of Means of yield, yield-componants and phenological traits in rapeseed cultivars*

\begin{tabular}{|c|c|c|c|c|c|c|c|c|c|c|c|c|}
\hline $\begin{array}{c}\frac{3}{3} \\
\frac{3}{2} \\
2 \\
3 \\
3 \\
3 \\
3 \\
3 \\
3\end{array}$ & 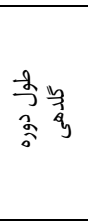 & $\begin{array}{l}\frac{3}{2} \\
2 \\
2 \\
i \\
3 \\
3 \\
5 \\
3 \\
3 \\
3 \\
3\end{array}$ & 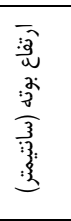 & $\begin{array}{l}\hat{3} \\
\hat{3} \\
\hat{2} \\
\hat{y}^{2}\end{array}$ & $\begin{array}{l}\frac{3}{3} \\
\frac{3}{3} \\
\frac{3}{3} \\
2 \\
\frac{y}{9} \\
\frac{9}{9}\end{array}$ & $\frac{3}{3} \frac{2}{3}$ & $\begin{array}{l}\frac{3}{3} \\
\frac{3}{2} \\
\frac{y}{2} \\
\frac{y}{9}\end{array}$ & 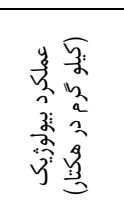 & $\begin{array}{l}.3 \\
.3 \\
\frac{2}{2} \\
\frac{1}{3} \\
\frac{1}{2} \\
a \\
a\end{array}$ & 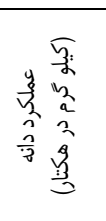 & $\begin{array}{l}\dot{\vec{a}} \\
: \vec{b}\end{array}$ & $\hat{.}^{\circ}$ \\
\hline $1 \% \mathrm{~F}^{\mathrm{bc}}$ & $r \varphi^{g}$ & $11 .^{b}$ & $a r^{b}$ & $\mathrm{~F} / \mathrm{r}^{\mathrm{ab}}$ & $r v^{a}$ &.$/\left.r r\right|^{\mathrm{a}}$ & $r .{ }^{\mathrm{de}}$ & $1.99 \mathrm{~N} / \mathrm{A}^{\mathrm{b}}$ & $r / \Lambda^{\mathrm{ab}}$ & $r r \Delta \Lambda^{a}$ & RGS003 & 1 \\
\hline 're & $M \mathrm{~h}^{\mathrm{h}}$ & $\| F^{\mathrm{a}}$ & $|A|^{\mathrm{b}}$ & $\left.r N\right|^{b}$ & $r \cdot{ }^{c}$ &.$/ r S \Lambda^{b}$ & $r \varepsilon \Lambda^{b c d}$ & $1 \cdot 1 V \pi / r^{b c}$ & $r / r^{\mathrm{bcd}}$ & $r q 1 q^{b c d}$ & RG405/03 & r \\
\hline||$^{c}$ & ${ }^{\mathrm{C}}$ & $9 r^{\mathrm{e}}$ & $199^{\mathrm{b}}$ & $F r / r^{\mathrm{ab}}$ & $r r^{a}$ &.$/ r r^{b}$ & $r r^{\mathrm{bcd}}$ & $1 \cdot \Delta F F / \kappa^{\mathrm{bc}}$ & $r / q^{c d}$ & Trefe & Kimberley & $r$ \\
\hline Irrc & $r \Lambda^{f}$ & $1.4^{c}$ & $119^{b}$ & $f i / v^{a b}$ & $r \Lambda^{\mathrm{a}}$ & $\cdot / r \Delta F^{b c}$ & $\mid q Y^{\mathrm{e}}$ & $q \& \mathrm{qv} / \mathrm{r}^{\mathrm{c}}$ & $r / r^{\mathrm{bcd}}$ & $M F \cdot \varphi^{c d e}$ & RGS006 & r \\
\hline $\mid r e^{b c}$ & $\mu^{e}$ & $1 \cdot r^{\mathrm{d}}$ & $u v^{b}$ & $\kappa \epsilon / \Delta^{\mathrm{a}}$ & $r F^{b}$ & . $/ T \Delta \Gamma^{b c c}$ & Trefede & $q \Delta \wedge \uparrow / \mu^{c}$ & $r / c^{\mathrm{bcd}}$ & $r F \cdot \gamma^{\text {cde }}$ & RGAS0324 & $\Delta$ \\
\hline $\mid k r^{\mathrm{ab}}$ & $r r^{\mathrm{f}}$ & $110^{\mathrm{a}}$ & $1 \mathrm{Fe}^{\mathrm{b}}$ & $F / \Delta^{a b}$ & $r^{b}$ & $\cdot / \pi F r^{\mathrm{C}}$ & ro. ${ }^{\text {be }}$ & $V \in \mathscr{Q V} / q^{d}$ & $r / \gamma^{\mathrm{de}}$ & ivare & Amica & 9 \\
\hline Ifrab & $\mathrm{kq} \mathrm{q}^{\mathrm{a}}$ & $9 r^{e}$ & $i v e^{b}$ & $\kappa \mu / V^{\mathrm{a}}$ & $r^{b c}$ & $\cdot / r \Delta \omega \gamma^{b c}$ & $r .^{\mathrm{de}}$ & $\Lambda v \& q / \Lambda^{c d}$ & $r / v^{\mathrm{abc}}$ & $r e q \gamma^{\text {cde }}$ & RG4403 & $v$ \\
\hline $1 \times 9^{a}$ & $\mu^{c d d}$ & $110^{\mathrm{a}}$ & $r q r^{\mathrm{a}}$ & $r N / K^{c}$ & $r^{d}$ & . $/ \pi \Psi^{d}$ & $r \omega \Delta^{a}$ & $\Lambda \Delta s \mathrm{~V} / q^{\mathrm{cd}}$ & $r / 1^{\mathrm{bcd}}$ & $r \cdot 1 K^{\mathrm{de}}$ & S-83 & $\wedge$ \\
\hline Ifrab & $e v^{a}$ & $q r^{\mathrm{e}}$ & $W \mathcal{F}^{\mathrm{b}}$ & $r \cdot / r^{\mathrm{ab}}$ & $r r^{b c}$ & . & $r \Delta \cdot{ }^{b}$ & $9 \Delta \& \Delta / \Lambda^{c}$ & $r / \Lambda^{e}$ & $194 \gamma^{\mathrm{de}}$ & Hysun110 & 9 \\
\hline$I r^{b c}$ & $r \Lambda^{b}$ & $9 r^{e}$ & $19 .{ }^{b}$ & $\kappa r / \omega^{\mathrm{ab}}$ & $r r^{b}$ & $\cdot / \Gamma \Delta F^{b c}$ & $r r^{b c}$ & ११४४//ด & $r / r^{\mathrm{bcd}}$ & $r r g v^{\text {cde }}$ & RG405/02 & 1. \\
\hline $1 \notin \varepsilon^{\mathrm{a}}$ & $r r^{\mathrm{de}}$ & $\| F^{a}$ & $19 r^{b}$ & $r q / a^{a b}$ & $r \cdot{ }^{c}$ & $\cdot / \pi \Delta \gamma^{b c}$ & $r \mu \mu^{\mathrm{de}}$ & $q \vee V / / \Delta^{c}$ & $r / \xi^{\mathrm{de}}$ & rrsqade & Sarigol & 11 \\
\hline$\pi \Lambda^{\mathrm{d}}$ & $\mu^{c d d}$ & $9 r^{e}$ & $1 v \Delta^{b}$ & $\kappa_{\alpha} / \mathrm{r}^{\mathrm{a}}$ & $r r^{a}$ & $. / \mu / f^{a}$ & $r \Delta \gamma^{b c}$ & $\| \varepsilon \mid V / F^{\mathrm{a}}$ & $r / q^{a b}$ & rrrqab & Hyola401 & ir \\
\hline$M \Lambda^{b c}$ & r & $90^{\mathrm{e}}$ & $1 v g^{b}$ & $r N / v^{a b}$ & $r^{b}$ & . /Rarbc & $r \Lambda^{\text {cde }}$ & $\Lambda \Delta \& F / T^{\mathrm{Cd}}$ & $r / \Lambda^{\mathrm{bcd}}$ & $r \Delta \wedge \varepsilon^{c d}$ & Hyola308 & سו \\
\hline $1 \notin \varepsilon^{b}$ & $r r^{c d}$ & $11 \pi^{\mathrm{ab}}$ & $1 \mathrm{Ve}^{\mathrm{b}}$ & $f i / /^{a b}$ & $r r^{b}$ &.$|r F|^{\mathrm{C}}$ & $r \in \Delta^{\text {be }}$ & $\Lambda F r v / q^{c d}$ & $r / g^{\mathrm{de}}$ & $r F \Delta \Lambda^{c d e}$ & Hyola60 & if \\
\hline 吾c & $\mu^{e}$ & $1 . r^{d}$ & $1 A v^{b}$ & $\kappa \epsilon / \Delta^{a}$ & $r r^{b}$ & $\cdot / r \Delta r^{b c}$ & Treq & $q \Delta \wedge \uparrow / \mu^{c}$ & $r / \kappa^{\mathrm{bcd}}$ & $r \mid q^{\text {cde }}$ & $\mathrm{PF}$ & 10 \\
\hline$|f|^{\mathrm{ab}}$ & $r r^{\mathrm{f}}$ & $110^{\mathrm{a}}$ & $i v e^{b}$ & $f \backslash / \Delta^{a b}$ & $r r^{b}$ &.$/ M F Y^{C}$ & $r \Delta .^{\text {be }}$ & $v \in \mathcal{E} V / q^{d}$ & $r / q^{\mathrm{de}}$ & $r \cdot q r^{\mathrm{de}}$ & Option500 & 19 \\
\hline $\mid r \cdot^{c}$ & r & $9 \Lambda^{\mathrm{de}}$ & $\mid w^{b}$ & $r \mathrm{~V} / \mathrm{a}^{\mathrm{ab}}$ & $r^{\mathrm{bc}}$ & $\cdot / \pi \Delta \Lambda^{b c}$ & $\left\langle\left. r\right|^{\text {cde }}\right.$ & $\Lambda F \Lambda \Delta / r^{c d}$ & $r / v^{\mathrm{bcd}}$ & $r a r \varepsilon^{c d}$ & Hyola420 & IV \\
\hline
\end{tabular}


با توجه به اينكه شاخص برداشت يكى از مهردمرترين

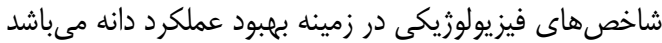

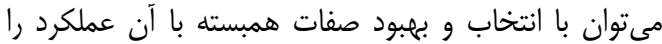

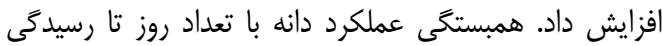

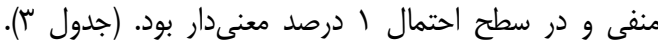

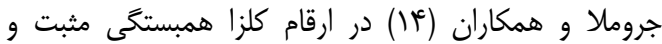

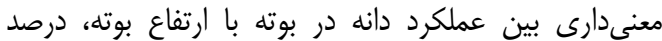

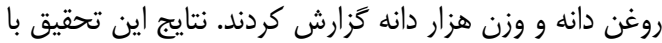

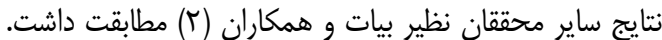

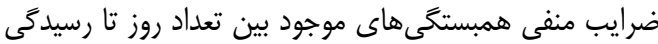

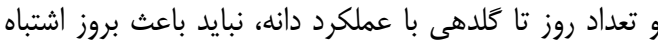

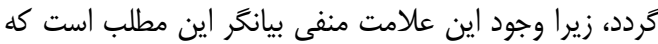

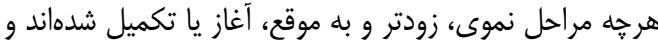

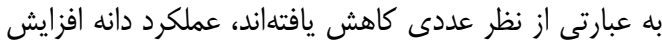

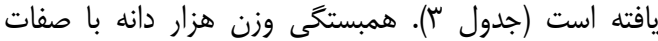

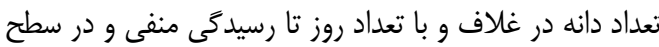

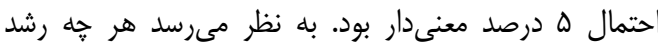

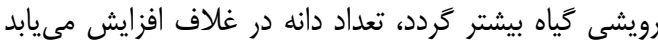

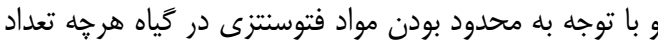

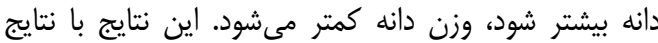
ساير محققان نظير بيات و همكاران (T) مطابقت داشت دانت

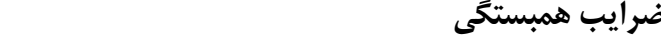

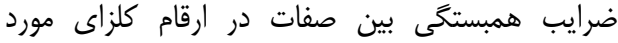

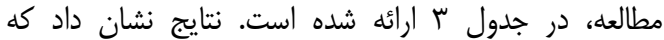

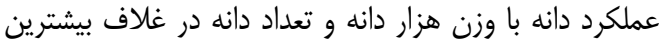

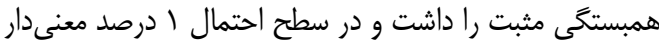

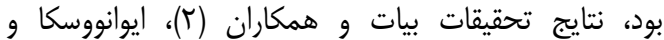

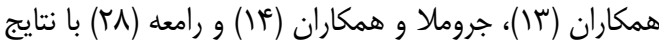

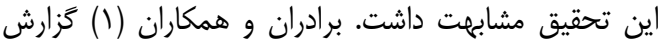

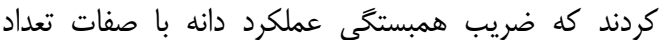

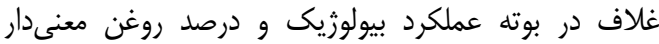

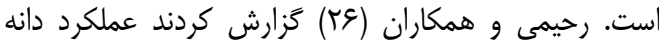

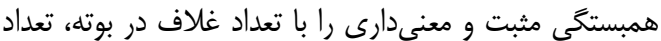

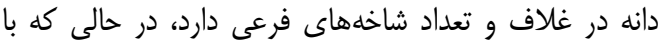

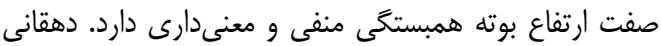

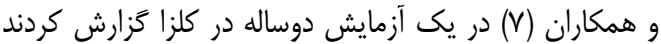

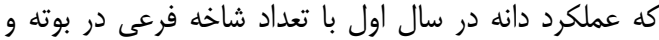

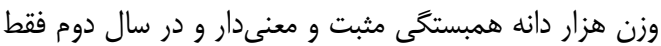

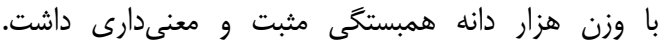

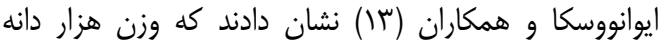

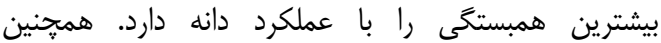

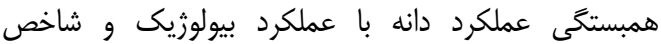
برداشت مثبت و در سطح احتمال ه درصد معنى معنى دار بود (جدول

جدول ץ- ضرايب همبستخى بين صفات مورد بررسى در IV رقم بهاره كلزا Table 3. Correlation coefficient among the characteristics in 17 rapeseed cultivars

\begin{tabular}{|c|c|c|c|c|c|c|c|c|c|c|}
\hline 1. & 9 & $\Lambda$ & $v$ & 9 & $\Delta$ & f & r & $r$ & 1 & صفات \\
\hline & & & & & & & & & 1 & ו- عملكرد دانه \\
\hline & & & & & & & & 1 & $\cdot|\Lambda|^{* * w}$ & ז- وزن هزار دانه \\
\hline & & & & & & & 1 &.$/ \cdot V$ &.$/ \% \Delta$ & ץ- تعداد غاف در بوته \\
\hline & & & & & & 1 & $-\cdot|r|$ & $-\cdot / \Delta V^{*}$ & $\cdot / \mathrm{V} / \mathrm{N}^{*}$ & f- تعداد دانه در غلاف \\
\hline & & & & & 1 &.$/ \cdot 1$ &.$- / \times q^{*}$ & r &.$- / T r$ & هـ درصد روغن \\
\hline & & & & 1 &.$- / 11$ & $-\cdot / q^{*}$ &.$/ 4 \gamma^{*}$ &.$- / 1 \Lambda$ &.$- / 41$ & و- ارتفاع بوته \\
\hline & & & 1 & Trt &.$- / T \Lambda$ & $-\cdot / T V$ & 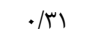 &.$- / 1 f$ &.$- / 1 T$ & V- تعداد روز تا كَلدهى \\
\hline & & 1 & $-\cdot / r \omega^{n s}$ & Tאוא. &.$/ 49$ & 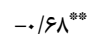 &.$/ 1 T$ & $-\cdot / \Delta T^{\prime \prime}$ & $-\cdot / 9 e^{\omega *}$ & ^- تعداد روز تا رسيدگى \\
\hline & 1 & $\cdot / \kappa^{\infty} \Delta$ &.$- / \% \Delta$ & $\cdot|r|$ & $\cdot / \cdot 1$ &.$- / 19$ &.$/ T \Lambda$ &.$- / 4 q^{*}$ & $-\cdot / \mathscr{A v}$ & ج- طول دوره گلدهى \\
\hline 1 &.$- / 11$ &.$- / \mu$ & $-\cdot / \mathcal{A P}$ & .1 .9 & - & $\cdot / T V$ &.$/ A T^{*}$ &.$/ 11$ & $\cdot|\omega|^{*}$ & • 1- عملكرد بيولوزيك \\
\hline.$- / 49$ & $-* / \mu f$ & $-\cdot / \Delta F^{*}$ & $\cdot / T V$ & זr/.- & $-\cdot|\Delta|^{*}$ &.$/ 41$ & (זם & $\cdot \mid \Delta T^{*}$ & $\cdot \mid \Delta \Lambda^{*}$ & 1 - شاخص برداشت \\
\hline
\end{tabular}

تا رسيدگى منفى و در سطح ه درصد معنىدار بود (جدول س).

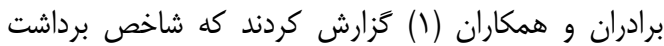

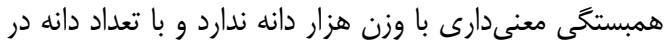

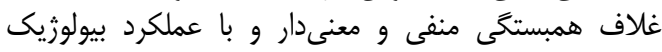

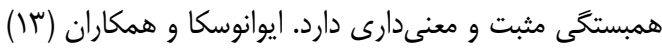

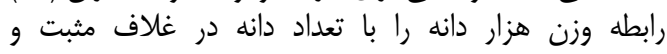

وزن هزار دانه با ساير صفات همبستخى مثبت و ناجيزى

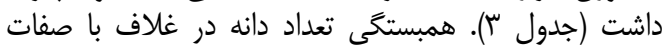

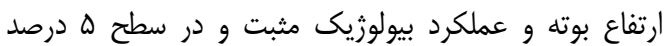

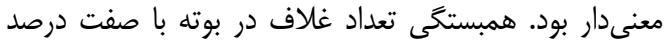

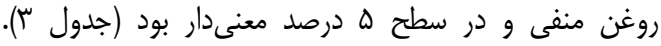
همبستخى شاخص برداشت با صفات درصد روغن و تعداد روز درو درد 
دانه را توجيه كرد. در مطالعات مشابهى، نتايج تجزيه

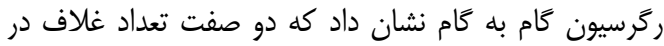

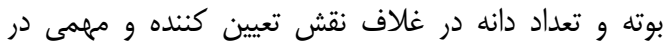

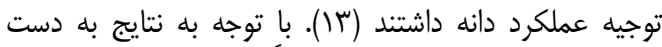

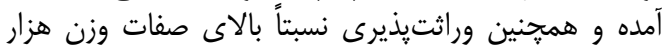

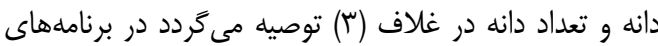

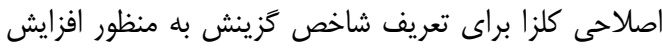
عملكرد دانه از اين صفات استفاده كَردد. تجزيه عليت

به منظور درك بهتر و تفسير دقيقتر نتايج به دست آمده

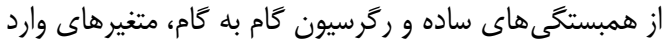

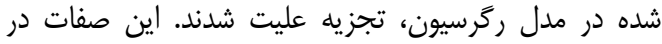

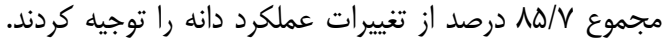

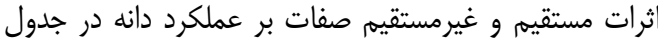

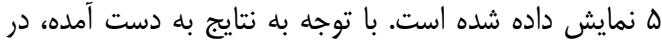

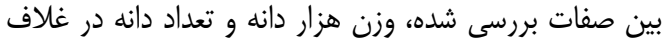

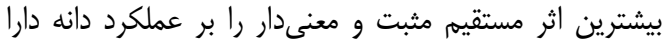

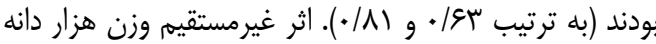

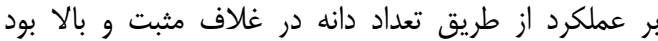

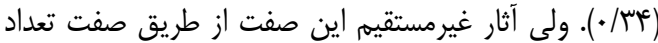

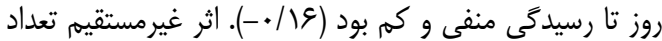

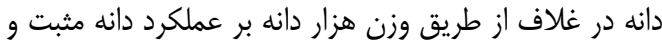

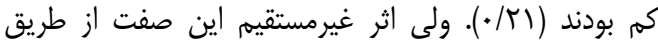

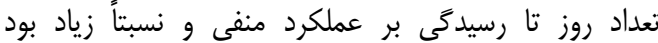

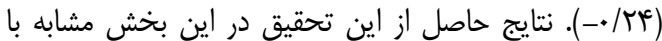

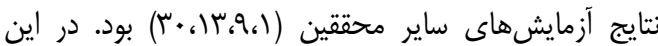

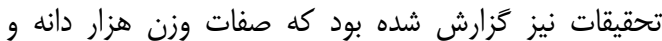

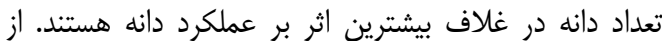

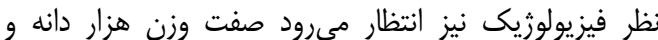

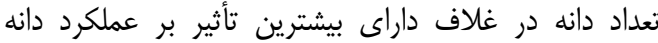

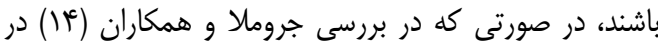

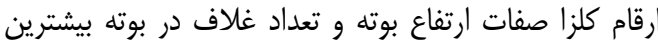

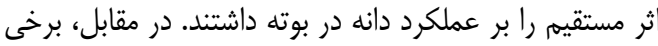

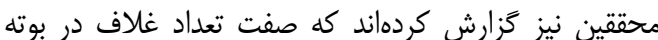

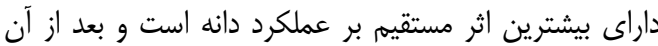

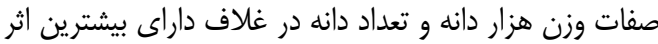

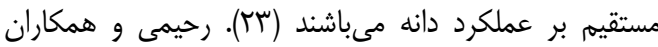

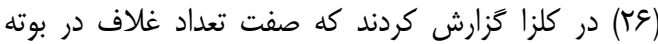

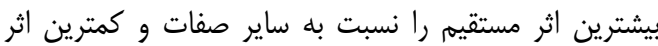
غيرمستقيم را از طريق صفات ديكر بر روى عملكرد دانه دارد.

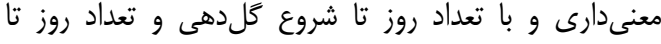

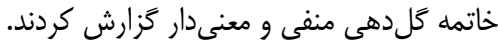

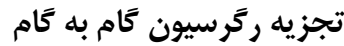

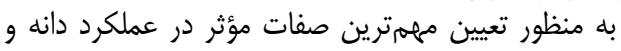

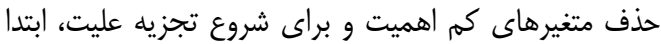

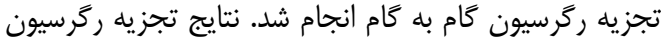

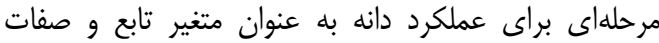

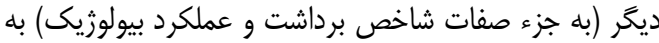

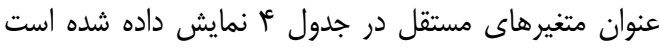

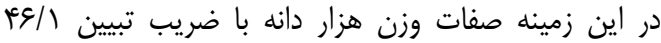

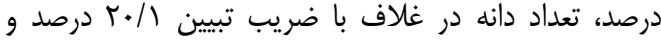

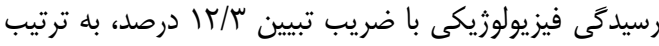

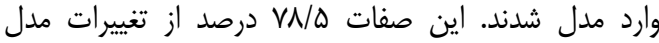

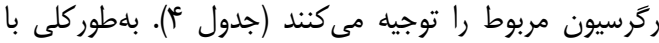

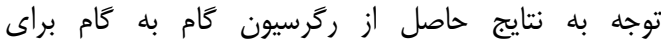

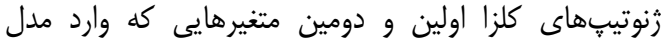

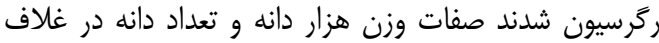

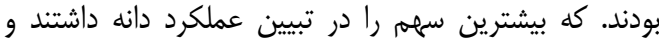

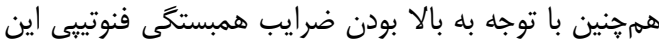

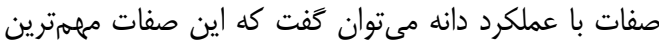

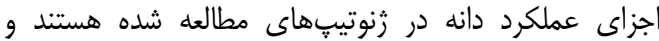

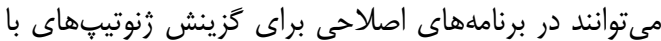

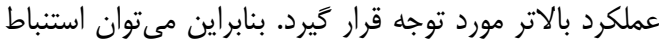

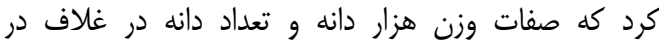

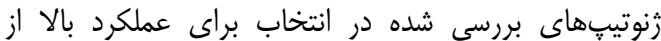

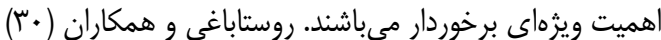

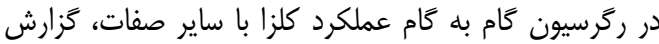

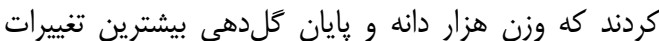

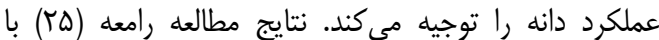

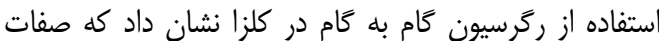

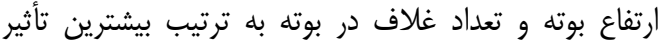

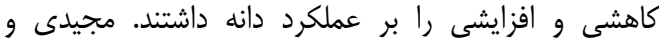

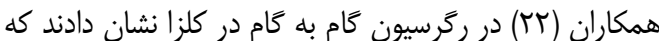

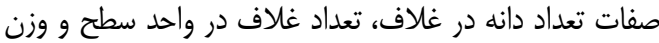

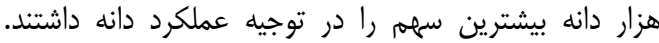

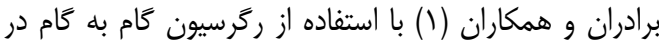

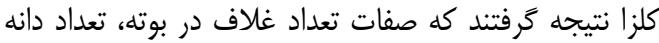

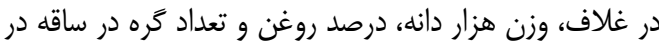

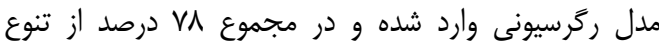

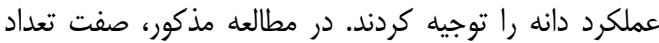
غلاف در بوته به تنهايى بخش كردي عمداى ان از تغييرات عملكرد 
جدول عا- نتايج رَرسيون گام به كام براى عملكرد دانه به عنوان متغير وابسته و ساير صفات به عنوان متغير مستقل Table 4. The results of stepwise regression analysis for seed yield as the response variable and yield-related traits as pridictor variables

\begin{tabular}{|c|c|c|c|c|c|}
\hline $\mathrm{R}^{r}$ & $\mathrm{~F}$ & خطاى استاندارد & ضريب ركر سيون جزء & متغير وارد شده & مر بله ورود متغير \\
\hline . & $\mid F r / 1 D^{* * 3}$ & $F T / T$ &.$/ 491$ & وزن هزار دانه & 1 \\
\hline . I99T & $q \mu / v^{* *}$ & $T Y / D)$ & $\cdot / r \cdot 1$ & تعداد دانه در غلاف & r \\
\hline ./VAD & $19 / 0^{*}$ & ro/sA & אזו/. & رسيدگى فيزيولوزيكى & $r$ \\
\hline
\end{tabular}

جدول ه- آثار مستقيم (روى قطر) و غيرمستقيم وزن هزار دانه، تعداد دانه در غلاف و تعداد روز تا رسيدگى بر عملكرد دانه كلزا Table 5. Direct (ondiagonal) and indirect effects of 1000-seed weight, number of seeds per pod and days to

\begin{tabular}{|c|c|c|c|c|c|}
\hline اثرات باقيمانده & ضريب همبستخى با عملكرد & تعداد روز تا رسيدىى & تعد غلاد دانه & وزن هزار & \\
\hline & $\cdot|\Lambda|^{* * 3}$ &.$- / 19$ & $\cdot / \mu F$ &. $\mid q^{4}$ & وزن هزار دانه \\
\hline & $\cdot / \mathrm{V} \mathrm{N}^{* * m}$ & $-\cdot / \pi r$ & 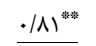 & $\cdot / \pi 1$ & تعداد دانه در غلاف \\
\hline ع & $-\cdot|9|^{* * a x}$ & $-\cdot / \Gamma \Delta^{*}$ &.$- / 14$ & $-\cdot / / V$ & تعداد روز تا رسيدگى \\
\hline
\end{tabular}

صفات وزن هزار دانه و تعداد دانه در غافل (أ) مىتوان در أناف

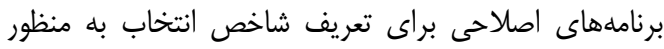
افزايش عملكرد دانه كلزا، از اين صفات استفاده گرددد.

هزينههاى مربوط به اجراى اين تحقيق از محل إنى اعتبارات

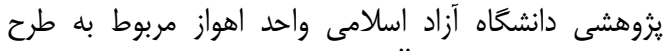

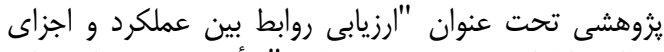

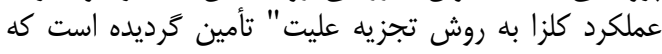
بدينوسيله تشكر مى كرد درد

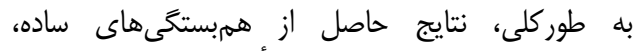

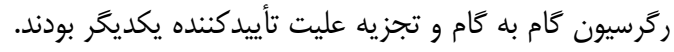

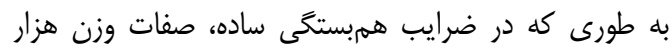

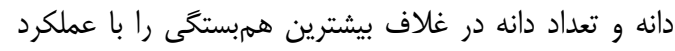

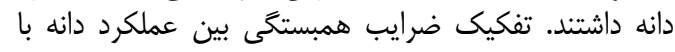

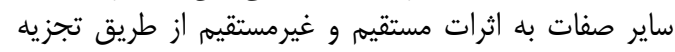

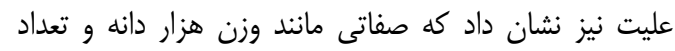

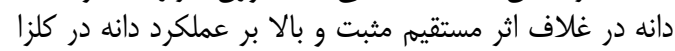

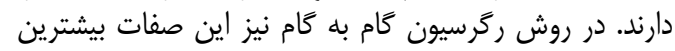

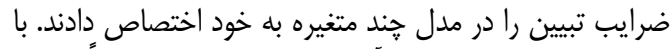

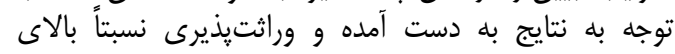

1. Baradaran, R., E. Majidi, F. Darvish and M. Azizi. 2006. Study of correlation relationships and path coefficient analysis between yield and yield components in rapeseed (Brassica napus L.). Journal of Agricultural Sciences, 12: 811-819.

2. Bayat, M., B. Rabiei, M. Rabiee and A. Moumeni. 2008. Assessment of relationship between grain yield and important agronomic traits of rapeseed as second culture in paddy fields. Journal of Crop production and processing, 12(45): 475-486 (In Persian).

3. Brandle, J.K. and P.B.E. Mcvetty. 1989. Heterosis and combining ability in hybrids derived from oilseed rape cultivars and inbred lines. Crop Science, 29: 1191-1195.

4. Clark, J.M. and G.M. Simpson. 1978. Growth analysis of Brassica napus. Canadian Journal of Plant Science 58: 587-597.

5. Darroch, A.B. and R.J. Baker. 1995. To measures of grain filling in spring wheat. Crop Science 35: 164-167.

6. Duarte, R.A. and M.W. Adams. 1972. Apath coefficient analysis of some yield component interrelations in field beans (Phaseolus vulgaris L.). Crop Sciense, 12: 579-582.

7. Dehghani, H., H. Omidi and N. Sabaghnia. 2008. Graphic analysis of relation of rapeseed using the biplot method. Agronomy Journal, 10: 143-149.

8. Farshadfar, A. 1996. Principle and multivariate methods. Razi University press. Kermanshah. Iran (In Persian).

9. Guo, J.C., X.X. Guo and R.H. Liu. 1987. A study of correlations between yield components in mutants of Brassica napus L. Oil Crops of China, 2: 23-25.

10. Guertin, W.H. and J.P. Bailey. 1982. Introduction to Modern Factor Analysis. Edwards Brothers Inc., Michigan. 
11. Hamza, S., W.B. Hamida, A. Rebai and M. Harrabi. 2004. SSR-based genetic diversity assessment among Tunisian winter barley and relationship with morphological traits. Euphytica, 135: 107-118.

12. Harman, H.H. 1976. Modern Factor Analysis, Third Edition Revised, University of Chicago Press.

13. Ivanovska, S., C. Stojkovski, Z. Dimov, A. Marjanovic-Jeromela, M. Jankulovska and L. Jankuloski. 2007. Interrelationship between yield and yield related traits of spring canola (Brassica napus L.) genotypes. Genetika, 39: 325-332.

14. Jeromela, A.M., R. Marinkovic., A. Mijic, Z. Zdunic and M. Jankulovska. 2008. Correlation and path analysis of quantitative traits in winter rapeseed (Brassica napus L.). Agriculturae Conspectus Scientificus, 73(1): 13-18.

15. Johnson, R.A. and D.W. Wichern. 1992. Applied multivariate statistical analysis. $3^{\text {rd }}$ ed. Englewood Cliffs, NJ: Prentice Hall, 767 pp.

16. Kakaei, M., A. Zebarjadi, A. Mostafaie and A. Rezaeizad. 2014. Genetic variation and traits interrelationship in some rapeseed genotypes using multivariate techniques under two moisture conditions. Journal of Applied Crop Breeding, 2(1): 31-45 (In Persian).

17. Khan, F.A., S. Ali, A. Shakeel, A. Saeed and G. Abbas. 2006. Correlation analysis of some quantitative characters in Brassica napus L. Journal of Agricultural Research, 44: 7-14.

18. Leilah, A.A. and S.A. Al-Khateeb. 2005. Yield analysis of canola (Brassica napus L.) using some statistical procedures. Saudi Journal of Biological Sciences, 12: 103-112.

19. Majidi, M.M., M. Jafarzadeh-Ghahdarijani, F. Rashidi and A. Mirlohi. 2016. Relationship of different traits in rapeseed (Brassica napus L.) cultivars under normal and drought conditions. Journal of Crop Breeding, 8(17): 55-65 (In Persian).

20. Manly, B.F.J. 2004. Multivariate Statistical Methods a Primer. $3^{\text {rd }}$ ed., Chapman \& Hall/CRC Inc., $226 \mathrm{pp}$.

21. Majidi M.M., M. Jafarzadeh-Ghahdarijani, F. Rashidi and A. Mirlohi. 2016. Relationship of different traits in rapeseed (Brassica napus L.) cultivars under normal and drought conditions. Journal of Crop Breeding, 8(17): 55-65 (In Persian).

22. Mohammadi S.A. and B.M. Prasanna. 2003. Analysis of genetic diversity in crop plants salient statistical tools and considerations. Crop Science, 43: 1235-1248.

23. Murat, T. and C. Vahdettin. 2007. Relationships between yield and some yield components in rapeseed (Brassica napus L.) cultivars by using correlation and path analysis. Pakistan Journal of Botany, 39: 81-84.

24. Naderi R. and M. Toorchi. 2012. Path analysis of the relationships between yield and some related traits in canola (Brassica napus L.) under salinity stress conditions. Annals of Biological Research, 3 (4): 1731-1734.

25. Naseri, F. 1990. Oil seed crops. Astan-ghods razavi press. Mashhad. Iran. 823 pp (In Persian).

26. Rahimi, M., M. Ramezani, and A. Ozoni Davaji. 2016. Investigation of path and correlation analysis of pattern and plant densities effect on two rapeseed cultivars. Journal of Crop Breeding, 8(19): 218227 (In Persian).

27. Ramee, V. 2012. Correlation and factor analyses of quantitative traits in rapeseed (Brassica napus L.). Agriculture Innovations and Research, 1(1): 19-73.

28. Rameeh, V. 2013. Multivariate analysis of some important quantitative traits in rapeseed (Brassica napus L.) advanced lines. Journal of Oilseed Brassica, 4(2): 75-82.

29. Rameeh, V. 2014. Multivariate regression analyses of yield associated traits in rapeseed (Brassica napus L.) genotypes. Advances in Agriculture, 2014. Article ID 626434, 5 pages.

30. Roostabaghi, B., H. Dehghan, B. Alizadeh and N. Sabaghnia. 2013. Study of diversity and evaluation of relationships between yield and yield components of rapeseed via multivariate methods. Journal of Crop production and processing, 2(6): 53-63 (In Persian).

31. Sabaghnia, N., H. Dehghani, B. Alizadeh and M. Moghaddam. 2010. Interrelationships between seed yield and 20 related traits of 49 canola (Brassica napus L.) genotypes in non-stressed and waterstressed environments. Spanish Journal of Agricultural Research, 8: 356-370.

32. Safari, S. and A.A. Mehrabi. 2016. Genetic relationships of rapeseed cultivars revealed by RAPD markers. Journal of Crop Breeding, 8(19): 170-177 (In Persian).

33. Sana, M., A. Ali, M.A. Malik, F.M. Saleem and M. Rafiq. 2003. Comparative yield potential and oil contents of canola cultivars (Brassica napus L.). Pakistan Journal of Agronomy, 2(1): 1-7.

34. Seiler, G.J. and R.E. Stafford. 1979. Factor analysis of components of yields in guar. Crop Science, 25: $905-908$

35. Sharma, S. 1996. Applied multivariate techniques. 1nd ed. John Wiley and Sons, New York. 493 pp.

36. Vafaei, S.N., A. Tobeh, A. Taee and S. Jamaati-e-Somarin. 2010. Study of phenology, harvest index, yield, yield components and oil content of different cultivars of rain-fed safflower. World Applied Science Journal, 8: 820-827. 


\title{
Evaluation of the Relationships among Yield and Related Traits in Spring Canola Cultivars using Path Analysis
}

\author{
Mahdi Soltani Howyzeh ${ }^{1}$, Mohammad Moradi ${ }^{2}$, Tayeb SakiNejad ${ }^{3}$, Saeed ZakerNejad $^{3}$ \\ and Adel Etaa
}

\author{
1- Department of Plant Breeding, Ahvaz Branch, Islamic Azad University, Ahvaz, Iran \\ (Corresponding author:soltani.m@iauahvaz.ac.ir) \\ 2- Department of Plant Breeding, Shoushtar Branch, Islamic Azad University, Shoushtar, Iran \\ 3- Department of Agronomy, Ahvaz Branch, Islamic Azad University, Ahvaz, Iran \\ Received: July 2, $2017 \quad$ Accepted: Non 13, 2016
}

\begin{abstract}
Performance of any breeding program is mainly due to the correlation among yield and relative importance of each component. This study was conducted to analysis the correlation among grain yield and some important traits in seventhin spring canola (Brassica napus L.) cultivars at the farm in safiabad, from 2014 to 2015. A randomized complete blocks design with four replications was used. The analysis of variance results indicated highly significant differences in canola genotypes for all studied traits, that it can indicate genetically variation be utilized for the effective selection for improving grain yield. Mean comparision showed that RGS003 and Hayola401 cultivars had higher grain yield than the other cultivars. The correlation coefficients among the grain yield and 1000-seed weight, number of seed per pod, HI and days to maturity were positive and significant. Results of stepwise regression analysis revealed that 1000-grain weight, number of pods per plant and days to maturity had significantly effects on grain yield. Path coefficient analysis revealed that the number of seed per pod and 1000-seed weight had the largest direct effects on grain yield, its seams possible to be use as selection criteria in breeding programs for improving grain yield of spring rapeseed cultivars.
\end{abstract}

Keywords: Correlation, Stepwise regression, Path analysis, Grain yield, Canola 